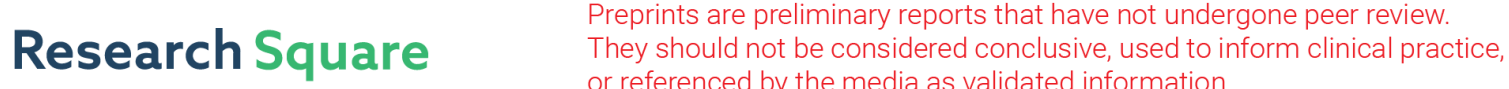 or referenced by the media as validated information. \\ Four-band non-Abelian topological insulator and its experimental realization
}

\section{Tianshu Jiang}

The Hong Kong University of Science and Technology

\section{Qinghua Guo}

Hong Kong University of Science \& Technology

\section{Ruo-Yang Zhang}

The Hong Kong University of Science and Technology

\section{Zhao-Qing Zhang}

Hong Kong University of Science \& Technology

Biao Yang ( $\sim$ biaoyang@ust.hk)

The Hong Kong University of Science and Technology https://orcid.org/0000-0002-5839-7261

Che Ting Chan

Department of Physics, The Hong Kong University of Science and Technology https://orcid.org/00000002-9335-8110

\section{Article}

Keywords: non-abelian topological insulator, band systems

Posted Date: July 1st, 2021

DOl: https://doi.org/10.21203/rs.3.rs-671793/v1

License: (c) (1) This work is licensed under a Creative Commons Attribution 4.0 International License.

Read Full License 


\title{
Four-band non-Abelian topological insulator and its experimental realization
}

Tianshu Jiang $^{1 \dagger}$, Qinghua Guo ${ }^{1 \dagger}$, Ruo-Yang Zhang ${ }^{1}$, Zhao-Qing Zhang ${ }^{1}$, Biao Yang ${ }^{1,2 *}$, C. T. Chan $^{1 *}$

${ }^{1}$ Department of Physics and Institute for Advanced Study, The Hong Kong University of Science and Technology, Hong Kong, China

${ }^{2}$ College of Advanced Interdisciplinary Studies, National University of Defense Technology, Changsha 410073, China

${ }^{\dagger}$ These authors contributed equally to this work.

*Correspondence to: yangbiaocam@,nudt.edu.cn; phchan@ust.hk;

\begin{abstract}
Very recently, increasing attention has been focused on non-Abelian topological charges, e.g. the quaternion group Q8. Different from Abelian topological band insulators, these systems involve multiple tangled bulk bandgaps and support non-trivial edge states that manifest the non-Abelian topological features. Furthermore, a system with even or odd number of bands will exhibit significant difference in non-Abelian topological classifications. Up to now, there is scant research investigating the even-band nonAbelian topological insulators. Here, we both theoretically explored and experimentally realized a four-band PT (inversion and time-reversal) symmetric system, where two new classes of topological charges as well as edge states are comprehensively studied. We illustrate their difference from four-dimensional rotation senses on the stereographically projected Clifford tori. We show the evolution of bulk topology by extending the 1D Hamiltonian onto a 2D plane and provide the accompanying edge state distributions following an analytical method. Our work presents an exhaustive study of four-band nonAbelian topological insulators and paves the way to other even band systems.
\end{abstract}

In mathematics, Abelian operators are commutative meaning that the result of two successive operations does not depend on the order in which they are written. If we focus on a single bandgap, topological physical systems ${ }^{1-6}$ are usually classified by Abelian groups, with the 
prime example being the ten-fold classification ${ }^{7,8}$ of Hermitian topological insulators and superconductors. Once multiple bandgaps are collectively considered, their coupling introduces richer physics that can make the classification non-Abelian ${ }^{9-13}$. A classic example is the quaternion group $Q_{8}=\{+1, \pm i, \pm j, \pm k,-1\}$ with $i^{2}=j^{2}=k^{2}=i j k=-1$, which has been used to classify the topological line defects in biaxial nematic liquid crystals ${ }^{14}$. Very recently, the non-Abelian group was used to describe the admissible nodal line configurations $^{12,15,16}$, Dirac/Weyl point braiding ${ }^{13,17,18}$ and intriguing triple nodal points ${ }^{19-21}$ in PT (inversion and time-reversal) symmetric systems. When more bands are involved, richer non-Abelian topological charges emerge ${ }^{9}$. Especially for the systems with even number of bands, several new classes of non-Abelian topological charges deserve special attention. A simple argument to this is that the even dimensional special orthogonal groups, i.e. $S O(2 N)$ with $N$ indicating positive integer, contain inversion symmetry, i.e. $-I_{2 N}$ (the negative $2 N \times 2 N$ identity matrix) .

\section{Non-Abelian topological charges in four-band models}

Here, for simplicity we focus on a four-band PT symmetric system. Choosing an appropriate basis the Hamiltonian can take real forms, i.e. $H(k)=H^{*}(k)$. When simultaneously considering all the three bandgaps between any two adjacent bands, the configuration space of the Hamiltonian is $M_{4}=O(4) / \mathbb{Z}_{2}^{4}$, with $O(4)$ being the $4 \mathrm{D}$ orthogonal group. This implies that the eigenstate frame remains intact under $O(4)$ rotation, while $\mathbb{Z}_{2}^{4}$ indicates each eigenstate has the gauge freedom of \pm 1 . The quantized charges that describe the underlying topology are found to be the non-Abelian based homotopy group ${ }^{9} \pi_{1}\left(M_{4}\right)=Q_{16}=\bigcup_{n_{i} \in\{0,1\}}\left\{ \pm e_{1}^{n_{1}} e_{2}^{n_{2}} e_{3}^{n_{3}}\right\}$, where $e_{1}, e_{2}, e_{3}$ are the basis of real Clifford algebra $C \ell_{0,3}$ with satisfying the relation $\left\{e_{i}, e_{j}\right\}=-2 \delta_{i j}$. There are 16 elements in the group and 10 conjugacy classes in total (see Table 1 as indicated by the curly braces). The group multiplication can be simply carried out with using the above relation, i.e. $\left(e_{1} e_{2}\right)\left(e_{1} e_{3}\right)=-e_{1} e_{1} e_{2} e_{3}=e_{2} e_{3}$. Although the labels with the Clifford algebra basis (see the $1^{\text {st }}$ column of Table 1) are convenient for group multiplication, it is not straightforward to decode the underlying physical meaning. In order to relate the charges to the rotations of the eigenstates, we rename all the charges one-to-one as shown in the 2 nd column of Table 1 . For example, we will see that $\pm q_{12}$ indicate that both the 1st and 2 nd bands acquire Zak phases of $\pi$ due to the rotation of their respective eigenvectors. Figure 1a shows the representative elements and their multiplication relations, the corresponding full multiplications are listed in Supplementary Tables 1 and 2. One may also 
notice that the paths (arrows) bridging two elements are not unique. It means the non-Abelian topological phase transitions are multiple-pathed, which is different from the single-pathed way in Abelian systems ${ }^{22}$.

In the following we study the topological properties of those charges. After topological band flattening, the mentioned PT symmetric four-band Hamiltonian can take the form of $H(k)=$ $R(k) I_{1234}(k) R^{T}$ with $R(k) \in S O(4)$ being the 4D special orthogonal group, $k \in[-\pi, \pi]$ being the first Brillouin zone (FBZ) and $I_{1234}=\operatorname{diag}(1,2,3,4)$. The Hamiltonian has four real eigenvectors as $H(k)|n\rangle=n|n\rangle$ with $n=1,2,3$ and 4 . When $k$ runs across the $\operatorname{FBZ}(k=$ $-\pi \rightarrow \pi)$, the rotation matrix $R(k)$ will continuously act on the eigenvector $|n\rangle$ and one finally obtains + or $-|n\rangle$ corresponding to the Zak phase of 0 or $\pi$, respectively. Without loss of generality, we assume $R(k=-\pi)=I_{4}$. Because $\operatorname{det}(R)=\lambda_{1} \lambda_{2} \lambda_{3} \lambda_{4}=1$ with $\lambda_{i}$ being the four eigenvalues of $R(k)$, it is easy to find there are exhaustively three categories of possibilities at $k=\pi$ : (1) All four $\lambda_{i}=1$; (2) Two $\lambda_{i}=1$, the other two $\lambda_{i}=-1$; (3) All four $\lambda_{i}=-1$.

The first category corresponds to two conjugacy classes $\{+1\}$ and $\{-1\}$. Although they are indistinguishable from the Zak phase description, charge +1 indicates the trajectories of eigenstate frame are contractible, while charge -1 indicates an non-contractible loop. Usually, charge -1 indicates that the eigenstate frame rotates $2 \pi$ in a rotation plane (or topologically equivalent configurations $)^{9,22}$. We will see their difference more explicitly by extending the $1 \mathrm{D}$ Hamiltonian onto a 2D plane (Figs. 1d and e); The second category consists of six conjugacy classes which can be distinguished using single band Zak phase arguments, regarding which two of the four bands have Zak phases of $\pi$; In the last category, all eigenstates flip their sign after $k$ runs across the 1D FBZ. This category originates from the inversion symmetry $\left(-I_{4}\right)$ mentioned above. The two group elements (classes) also share the same Zak phase distribution and are indistinguishable from the conventional Abelian arguments. Their difference is reflected in the sense of eigenstate rotation in four-dimension.

With setting $R(k)=\exp \left(\phi \sum_{i<j=1: 4} n_{i j} L_{i j}\right)$, we obtain the explicit form of the flat band Hamiltonian, where six skew-symmetric matrices $L_{i j}$ with entries $\left(L_{i j}\right)_{a, b=1: 4}=-\delta_{i a} \delta_{j b}+$ $\delta_{i b} \delta_{j a}$ span the basis of Lie algebra $\mathfrak{s D}(4), \phi(k)$ is the rotation angle and $n_{i j}(k)$ determines the rotation plane. For example, the Hamiltonian of charge $q_{12}$ can be given with $R(k)=$ 
$\exp \left(\frac{k+\pi}{2} L_{12}\right)$, while charge -1 can be obtained with $R(k)=\exp \left[(k+\pi) L_{12}\right]$. Except for the charges of $\pm q_{1234}$, the rest have their counterparts in the three-band systems ${ }^{22}$ studied previously. Thus, we mainly focus on the charges $\pm q_{1234}$ which are unique in the four-band models.

While the non-Abelian topological charges are defined on one-dimensional periodic lattices, their topological characters would be more straightforward to visualize after we generalize the 1D Hamiltonians onto a 2D extended plane, where each non-Abelian topological charge characterizing the 1D loop is reflected by the specific configuration of band degeneracies encircled by the 1D loop in the 2D plane. After trigonometrically expanding the Hamiltonian $H(k)$, we make the substitutions like $\cos k \rightarrow \rho \cos k=k_{1}$ and $\sin k \rightarrow \rho \sin k=k_{2}$ and show the corresponding two-dimensional bands in Figs. 1b-e. The original 1D Hamiltonian in $k$ space is a unit circle (white circles in Figs. 1b-e) in the 2D extended plane which encircles non-removable degeneracies explicitly exhibiting the underlying topological obstacles. The charge +1 (Fig. 1e), it is trivial as there is no degeneracy enclosed by the white circles, while for charges $\pm q_{m n}$ (Fig. 1b) and -1 (Fig. 1d) the 1D unit circles enclose linear and quadratic degeneracies, respectively. These $2 \mathrm{D}$ degeneracies will topologically contribute to edge/domain-wall states of the 1D systems, i.e. the linear/quadratic degeneracy implies one/two topologically protected edge states.

The charge $q_{1234}$ can be factorized as $q_{1234}=q_{12} q_{34}, q_{1234}=q_{14} q_{23}$ and $q_{1234}=-q_{13} q_{24}$ (the minus sign is induced by the odd permutation of subscripts). Note that the two factors are commutative, i.e. $q_{12} q_{34}=q_{34} q_{12}$, in nodal links which means all nodes formed by more distant (i.e. sharing no common band) pairs of bands commute ${ }^{9}$. In Fig. 1c we show the corresponding extended 2D band degeneracies of the three cases. They all belong to the same charge and thus can be continuously transformed into each other without closing the bandgap (see Figure 3 ). The charge $-q_{1234}$ shares the same two-dimensional band degeneracies with $q_{1234}$. Note that $\pm q_{1234}$ belong to two different conjugacy classes, which is one of the key points that fundamentally distinguishes them from the charges $\pm q_{m n}$. We will show their topological differences in the following section from the eigenstate rotation perspective.

We note that the nodal ring degeneracies in Figs. $1 \mathrm{~b}\left( \pm q_{14}\right)$ and $\mathrm{c}\left(-q_{13} q_{24}\right)$ are accidental in the flat band models, each will be split into linear Dirac cones in more general situations (see 
Figure 5b and Supplementary Figure 16c, respectively). Other triple degeneracies are similar to charges $\pm j$ in three-band models ${ }^{22}$, where there are three bands being involved. The fourfold degeneracy in Fig. 1c $\left(q_{14} q_{23}\right)$ is also admissible rather than stable here.

\section{Eigenstates on three-sphere $-S^{3}$}

Here, we illustrate rotation configurations pertaining to different charges of the generalized quaternion group $Q_{16}$. The normalized eigenstates of $H(k)$ are all real and can be parametrized by Hopf coordinates $(\alpha, \eta, \beta)$ on the three-sphere $-S^{3}$. Their four components can be written as $(u=\cos \alpha \sin \eta, x=\sin \alpha \sin \eta, y=\cos \beta \cos \eta, z=\sin \beta \cos \eta)$, where $\alpha$ and $\beta$ respectively correspond to the two rotation angles in the two orthogonal invariant planes as shown in Fig. 2a (also see Supplementary Information II: Rotations in four-dimension ${ }^{23-25}$ ), while $\eta$ determines the proportions projected onto the two planes. When $\alpha \neq 0, \beta=0$ (or $\alpha=$ $0, \beta \neq 0$ ), the rotations are called single rotations. For example, all ideal rotations $R(k)$ with $k=-\pi \rightarrow \pi$ enabling charge $q_{12}$ belong to the case with setting $\eta=\frac{\pi}{2}$ and $\alpha=\frac{k+\pi}{2}$, where "ideal" indicates the flat band model given above. Note that all general models can be continuously transformed into the ideal flat band model and they are topologically equivalent. Other charges including $\pm q_{m n}$ and -1 can be realized in a similar manner. It is easy to see that the eigenstates in one plane (i.e. oyz plane when $\eta=\frac{\pi}{2}$ ) can be fixed for these cases, while they rotate on the other orthogonal plane (i.e. oux plane). In other words, the ideal rotations can be carried out in a two-dimensional subspace. It is worth pointing out that in contrast on which plane the eigenstates rotate, the crucial property of these topological charges is that the trajectories of eigenstates cannot contract to isolated points. The difference of charges $\pm q_{m n}$ is reflected by which two bands (the mth and nth) are noncontractible, while charge -1 requires all four trajectories cannot contract simultaneously.

When both $\alpha \neq 0$ and $\beta \neq 0$, the rotations are dubbed as double rotations (Fig. 2a), where there are two possibilities: rotating on the two planes in the same $(\alpha \beta>0)$ or opposite $(\alpha \beta<$ 0 ) senses. The charges $\pm q_{1234}$ have to be realized with continuous double rotations, which means $R(k)$ at each $k$ point is a double rotation. Interestingly, when $\eta=\frac{\pi}{4}$, the parametric set $(u, x, y, z)$ constructs a Clifford torus ${ }^{26}$, which is the Cartesian product of two circles in $\mathbb{R}^{4}$ (e.g. $S_{A}^{1} \in$ oux,$S_{B}^{1} \in$ oyz and $S_{A}^{1} \times S_{B}^{1} \in \mathbb{R}^{4}$ ). The Clifford torus can be stereographically projected $^{26}$ into $\mathbb{R}^{3}$ as a conventional torus, i.e. $\left(\frac{x}{1-u}, \frac{y}{1-u}, \frac{z}{1-u}\right)$, on which we can pictorially 
illustrate the difference between charges $\pm q_{1234}$ from the rotation senses of eigenstate trajectories as shown in Fig. 2b. The two panels correspond to $\alpha=\beta=\frac{k+\pi}{2}$ (left, $q_{1234}$ ) and $\alpha=-\beta=\frac{k+\pi}{2}$ (right, $-q_{1234}$ ), respectively (see other cases in Supplementary Figure 5).

We further propose another orthographical projection method, which projects each 4D trajectory into 3D space from four orthogonal views. This is similar to the three-view drawing, which is the orthographic projection form 3D space to $2 \mathrm{D}$ plane. Take the first panel of Fig. 2c as an example, we plot the trajectories in the $x y z$ subspace so that it is an orthographic projection from the view of $u$ direction. Figures $2 \mathrm{c}$ and $\mathrm{d}$ respectively correspond to $+q_{1234}$ and $-q_{1234}$, where eigenstate trajectories are mapped onto four solid spheres in $\mathbb{R}^{3}$. One can see their only difference is that the rotation directions in the oux plane are opposite. Orthographical projections for other charges are listed in Supplementary Figures 1-4. In Fig. 2e, we show the topological phase transition between them, where there are inevitably two linear crossings between the first and second bands as system parameter $w_{A B}$ changes (without relying on a joint basepoint as they belong to different classes).

\section{Zak phases and evolution of edge states}

After understanding the non-Abelian topological charges from the perspective of eigenstate frame rotations, we now show their relations to the Zak phases of each band as well as edge/domain-wall states. In a PT-symmetric system, the Zak phases of each band will take the quantized values of 0 or $\pi$, which have been shown in Table 1, i.e. $\lambda_{i}=-1$ indicates Zak phase of $\pi$. We further refine the Zak phase of $\pi$ to be $\pm \pi$, where " \pm " is used to differentiate between charges $\pm q_{m n}$ (two elements in the same conjugacy class). All of the corresponding single band Zak phases are exhaustively summarized in Fig. 3a. For charges $\pm q_{m n}$, two corresponding bands with noncontractible eigenstate trajectories carry Zak phases of $\pm \pi$, and the bandgap sandwiched by them supports edge states at hard boundaries of a finite lattice. We take the case of $\pm q_{12}$ as an example as shown in Fig. 3b. Edge states of other $\pm q_{m n}$ charges are shown in the Supplementary Figure 6 . We label charge -1 with $2 \pi$, which indicates noncontractible $2 \pi$ rotation here ${ }^{22}$.

For charges $\pm q_{1234}$, two eigenstates rotate $\pi$, while the other two rotate $\pm \pi$ when $k=-\pi \rightarrow$ $\pi$, respectively. As shown in Fig. 1c, there are three ways of factorization. We further show them schematically in Fig. 3c, where each double-headed arrow represents one factorization. 
The commutative property between two factor charges, i.e. $q_{12} q_{34}=q_{34} q_{12}$, is implied by the double-headed arrows. The fact that $q_{12} q_{34}$ (type-I), $-q_{13} q_{24}$ (type-II) and $q_{14} q_{23}$ (type-III) are the same element in the group can be visualized by constructing a transformation between them without gap closing. The continuous transition between different factorizations can be explicitly parameterized. For example from $q_{12} q_{34} \rightarrow-q_{13} q_{24}$, we have $H(k)=$ $R_{2} R_{1} I_{1234} R_{1}^{-1} R_{2}^{-1} \quad$, with $R_{1}(k)=\exp \left[(k+\pi) / 2\left(\cos \theta_{I \rightarrow I I} L_{12}-\sin \theta_{I \rightarrow I I} L_{13}\right)\right] \quad$ and $R_{2}(k)=\exp \left[(k+\pi) / 2\left(\cos \theta_{I \rightarrow I I} L_{34}+\sin \theta_{I \rightarrow I I} L_{24}\right)\right]$ as shown in Fig. 3c (see the evolution of eigenstate trajectories in Supplementary Figure 3). In other words, the pair of two orthogonal invariant planes rotates with $\theta_{I \rightarrow I I}$. We further study the accompanying evolution of edge states at hard boundaries as shown in Figs. 3d-f. They have analytical results: $E^{ \pm}=$ $\frac{5}{2} \pm \frac{\sqrt{2}}{4} \sqrt{5+3 \cos 2 \theta_{I \rightarrow I I}}, E^{ \pm}=\frac{5}{2} \pm \frac{1}{2} \cos \theta_{I I \rightarrow I I I}$ and $E^{ \pm}=\frac{5}{2} \pm \sin \theta_{I I I \rightarrow I}$, respectively. Detailed analytical methods are provided in Supplementary Information IV: Analytical solutions of edge states for the flat-band models. There are a total of two edge states pumping between different bandgaps. Their field distributions are given in the Supplementary Figures 7-9. The existence of these edge modes can be inferred heuristically by examining the band degeneracies of the extended 2D model. In Figs. 3g-i, we show the radial cuts of their extended two-dimensional bands, where one can easily finds that each linear degeneracy point at $k_{r}=0$ implies the position of each edge state in Figs. 3d-f, respectively. Note that in these flat band cases, only the degeneracies at $k_{r}=0$ imply topological edge states, while other degeneracies $\left(k_{r} \neq 0\right)$ accidentally emerge from the 2D nodal rings (e.g. see Fig. 1c) which have no topological implication.

We also show the edge state evolution for charge -1 in Supplementary Figures 10 and 11 (see the analytical solutions in Supplementary Information IV). Along the 12 edges of the charge -1 octahedron (Supplementary Figure 10a), the evolution shows strong resemblance to the three-band models $\mathrm{s}^{22}$. It is because only three bands participate in the edge state pumping. As such, all these transitions can be understood via the rotations of eigenstates in the subgroup $S O(3)$, while the fourth band is fully fixed and decoupled. One other important note-there are 12 possible routes rather than 15 (naively from $C(6,2)=15$ ), because it is impossible to evolve directly between two orthogonal planes (or between the diagonal points linked by the dashed lines in Supplementary Figures $10 \mathrm{a}$ and b), e.g. between $q_{12}^{2}$ and $q_{34}^{2}$. We also find the transition can take arbitrary routes on the 8 faces of the charge -1 octahedron (see an example in Supplementary Figures 10d and 11). Supplementary Figure 12 shows the evolution of 2D 
extended band degeneracies, which help us to understand the pumping of edge states accordingly, e.g. the double-quadratic or triple-linear degeneracies at $k_{r}=0$ predict the emergence of topological edge states ${ }^{22}$.

\section{Observation of charges $\pm q_{1234}$ in a transmission line network}

In order to realize and characterize charges $\pm q_{1234}$, we designed a transmission line network $^{22,27,28}$ (see the sample photo in Supplementary Figure 13) consisting of 11 unit-cells. There are four meta-atoms A, B, C and D in one unit-cell. The real-space Hamiltonian reads (see details in Supplementary Information III),

$$
\mathcal{H}=\sum_{n}\left(\sum_{\substack{X=A, B, C, D \\ Y=A, B, C, D}} s_{X Y} c_{X, n}^{\dagger} c_{Y, n}+\sum_{\substack{X=A, B, C, D \\ Y=A, B, C, D}} v_{X Y} c_{X, n}^{\dagger} c_{Y, n+1}+\text { h. c. }\right)
$$

where $c_{X, n}^{\dagger}$ and $c_{X, n}$ are creation and annihilation operators on the sub-lattice ' $X / Y$ ' and site ' $n$ ', respectively. In order to realize an explicitly real Hamiltonian in momentum space, we introduce imaginary hoppings ${ }^{22}$. More details on the experimental realization are provided in Supplementary Information $\mathrm{V}$ and $\operatorname{ref}^{22}$.

The left two panels (BulkS) in Fig. 4a show respectively the numerically calculated and experimentally measured energy bands. We plot the corresponding eigenstate trajectories of the four bands in Figs. $4 \mathrm{c}$ and d. In the experimental model, at each $k$ we can see one rotation plane is spanned by the eigenvectors of the first and second bands and the other one by those of the third and fourth bands. We can expect that there are two topological edge states in total and one locates in the first bandgap (sandwiched by the first and second bands) while the other one in the third bandgap (formed by the third and fourth bands). The rightmost panels (EdgeS) of Fig. 4 a confirm our expectation. The detailed field distribution is provided in Supplementary Figure $14 \mathrm{~b}$. The distribution of edge states can also be directly inferred from the 2D extended energy bands as shown in Fig. 4b, where there is one linear Dirac cone between the first/third and second/fourth bands.

\section{Domain-wall states between charges $+q_{1234}$ and $-q_{1234}$}

If two samples with different non-Abelian topological charges meet at a domain-wall ${ }^{22}$, some domain-wall states (DMS) will emerge and their existence can be predicted by defining a "domain-wall charge" $\Delta Q=Q_{L} / Q_{R}$. Here $Q_{L}$ and $Q_{R}$ are the non-Abelian topological charges 
of the left and right samples, respectively. The quotient charge $\Delta Q$ is also an element of the non-Abelian group and governs the properties (including both location and number) of the DWS. We note that the appearance of the domain-wall charge -1 in the three-band system can only be well defined by assuming a joint $k$-space basepoint between the left and right samples ${ }^{22}$. Otherwise one cannot distinguish two non-Abelian topological charges (e.g. $+i$ and $-i$ ) in the same conjugacy class of a three-band system, and thus the domain-wall charge becomes illdefined. In the four-band system, however, there exists a basepoint-free domain-wall charge taking value of -1 between charges $+q_{1234}$ and $-q_{1234}$. It is because they belong to two different conjugacy classes.

In the experiment, we construct a domain-wall (blue spheres in Fig. 4e) between charges $\pm q_{1234}$ as shown in Fig. 4 e, where we flip the directions of imaginary hoppings between metaatoms $\mathrm{C}$ and $\mathrm{D}$ as denoted by the blue arrows to realize the charge $+q_{1234}$ at the righthand side of the domain wall. Figure $4 \mathrm{f}$ shows the domain-wall states between them, where the left inset is the simulated energy levels and the right two insets indicate the measured spectra on the domain-wall for two different excitation/probe locations accordingly. These results indicate that there are two nearly degenerate topological domain-wall states in the third bandgap. This is the same as the hard boundary edge states of charge -1 and thus confirms our prediction. The detailed field distribution is provided in Supplementary Figure 15.

\section{Observation of charges $\pm q_{14}$ in a transmission line network}

In addition, we also experimentally studied charges $\pm q_{14}$, which is also interesting in the fourband models as it exhibits three edge states in the three bandgaps. As shown in Fig. 5, from the bulk bands (Fig. 5a-BulkS), edge state distributions (Fig. 5a-EdgeS) and eigenstate trajectories (Fig. 5c-d), the numerical calculations correctly predict the experimental results. Different from Fig. $1 \mathrm{~b}\left( \pm q_{14}\right)$ of the flat band model, the 2D extended energy bands in Fig. $5 \mathrm{~b}$ are bridged by three linear Dirac cones. As mentioned above, each implies one edge state (per edge), as verified in Fig. 5a-EdgeS. For charge $\pm q_{14}$, there is no complete bandgap in the $2 \mathrm{D}$ extended bands. It can be regarded as the generalization of charge $\pm j$ in three-band models $\mathrm{s}^{9,22}$.

\section{Discussions and conclusion}

Other general configurations of charges $\pm q_{1234}$ are shown in Supplementary Figures 16 and 17 , corresponding to the factorizations of $-q_{13} q_{24}$ and $-q_{14} q_{23}$, respectively. As mentioned 
above, the ring degeneracy formed by the second and third bands in Fig. 1c $\left(-q_{13} q_{24}\right)$ splits into two Dirac cones as shown in Supplementary Figure 16c, which further imply two edge states (per edge) in Supplementary Figure 16d. The general model of charge -1 in Supplementary Figure 18 shows one triple linear degeneracy, being similar to what we have seen in the three-band models, as such the edge state distributions ${ }^{22}$.

Our exhaustive study of all non-Abelian topological charges of the PT symmetric four-band Hamiltonians will constructively stimulate the related research on 2D systems, e.g. twisted bilayer graphene $\mathrm{e}^{10,29,30}$. The PT symmetric systems also may contribute to exotic fragile topological states ${ }^{31}$ and even topological effective gravitational theory ${ }^{32}$. The studies can be easily transferred to other artificial platforms including optical lattices ${ }^{33}$, photonics $^{34-36}$ and phononics $^{37}$. 


\section{Reference:}

1 Hasan, M. Z. \& Kane, C. L. Colloquium: Topological insulators. Reviews of Modern Physics 82, 3045-3067, doi:10.1103/RevModPhys.82.3045 (2010).

2 Qi, X.-L. \& Zhang, S.-C. Topological insulators and superconductors. Reviews of Modern Physics 83, 1057-1110, doi:10.1103/RevModPhys.83.1057 (2011).

3 Ando, Y. \& Fu, L. Topological Crystalline Insulators and Topological Superconductors: From Concepts to Materials. Annual Review of Condensed Matter Physics 6, 361-381, doi:10.1146/annurev-conmatphys-031214-014501 (2015).

4 Chiu, C.-K., Teo, J. C. Y., Schnyder, A. P. \& Ryu, S. Classification of topological quantum matter with symmetries. Reviews of Modern Physics 88, 035005, doi:10.1103/RevModPhys.88.035005 (2016).

5 Armitage, N. P., Mele, E. J. \& Vishwanath, A. Weyl and Dirac semimetals in threedimensional solids. Reviews of Modern Physics 90, 015001, doi:10.1103/RevModPhys.90.015001 (2018).

6 Ozawa, T. et al. Topological photonics. Reviews of Modern Physics 91, 015006, doi:10.1103/RevModPhys.91.015006 (2019).

7 Schnyder, A. P., Ryu, S., Furusaki, A. \& Ludwig, A. W. W. Classification of topological insulators and superconductors in three spatial dimensions. Physical Review B 78, 195125, doi:10.1103/PhysRevB.78.195125 (2008).

8 Kitaev, A. Periodic table for topological insulators and superconductors. AIP Conference Proceedings 1134, 22-30, doi:10.1063/1.3149495 (2009).

9 Wu, Q., Soluyanov, A. A. \& Bzdušek, T. Non-Abelian band topology in noninteracting metals. Science 365, 1273, doi:10.1126/science.aau8740 (2019).

10 Ahn, J., Park, S. \& Yang, B.-J. Failure of Nielsen-Ninomiya Theorem and Fragile Topology in Two-Dimensional Systems with Space-Time Inversion Symmetry: Application to Twisted Bilayer Graphene at Magic Angle. Physical Review X 9, 021013, doi:10.1103/PhysRevX.9.021013 (2019).

11 Tiwari, A. \& Bzdušek, T. Non-Abelian topology of nodal-line rings in \$ \mathcal \{PT\} \$-symmetric systems. Physical Review B 101, 195130, doi:10.1103/PhysRevB.101.195130 (2020).

12 Yang, E. et al. Observation of Non-Abelian Nodal Links in Photonics. Physical Review Letters 125, 033901, doi:10.1103/PhysRevLett.125.033901 (2020). 
13 Bouhon, A. et al. Non-Abelian reciprocal braiding of Weyl points and its manifestation in ZrTe. Nature Physics, doi:10.1038/s41567-020-0967-9 (2020).

14 Mermin, N. D. The topological theory of defects in ordered media. Reviews of Modern Physics 51, 591-648, doi:10.1103/RevModPhys.51.591 (1979).

15 Wang, D. et al. Intrinsic in-plane nodal chain and generalized quaternion charge protected nodal link in photonics. Light: Science \& Applications 10, 83, doi:10.1038/s41377-021-00523-8 (2021).

16 Park, H., Wong, S., Zhang, X. \& Oh, S. S. Non-Abelian charged nodal links in dielectric photonic crystal. arXiv:2102.12546 [physics.optics] (2021).

17 Peng, B., Bouhon, A., Monserrat, B. \& Slager, R.-J. Non-Abelian braiding of phonons in layered silicates. arXiv:2105.08733 [cond-mat.mes-hall] (2021).

18 Jiang, B. et al. Observation of non-Abelian topological semimetals and their phase transitions. arXiv:2104.13397 [cond-mat.mes-hall] (2021).

19 Lenggenhager, P. M., Liu, X., Neupert, T. \& Bzdušek, T. Universal higher-order bulk-boundary correspondence of triple nodal points. arXiv:2104.11254 [condmat.mes-hall] (2021).

20 Guo, Q. et al. Observation of Three-Dimensional Photonic Dirac Points and SpinPolarized Surface Arcs. Physical Review Letters 122, 203903, doi:10.1103/PhysRevLett.122.203903 (2019).

21 Lenggenhager, P. M., Liu, X., Tsirkin, S. S., Neupert, T. \& Bzdušek, T. From triplepoint materials to multiband nodal links. Physical Review B 103, L121101, doi:10.1103/PhysRevB.103.L121101 (2021).

22 Guo, Q. et al. Experimental observation of non-Abelian topological charges and edge states. Nature 594, 195-200, doi:10.1038/s41586-021-03521-3 (2021).

23 Perez-Gracia, A. \& Thomas, F. On Cayley's Factorization of 4D Rotations and Applications. Advances in Applied Clifford Algebras 27, 523-538, doi:10.1007/s00006-016-0683-9 (2017).

24 Le Bihan, N. The geometry of proper quaternion random variables. Signal Processing 138, 106-116, doi:https://doi.org/10.1016/j.sigpro.2017.03.017 (2017).

25 Erdoğdu, M. \& Özdemir, M. Simple, Double and Isoclinic Rotations with a Viable Algorithm. Mathematical Sciences and Applications E-Notes, 8 (1), 11-24, doi:10.36753/mathenot.642208 (2020).

26 McCuan, J. \& Spietz, L. Rotations of the three-sphere and symmetry of the Clifford torus. arXiv:math/9810023 [math.MG] (1998). 
27 Zhao, E. Topological circuits of inductors and capacitors. Annals of Physics 399, 289313, doi:https://doi.org/10.1016/j.aop.2018.10.006 (2018).

28 Jiang, T. et al. Experimental demonstration of angular momentum-dependent topological transport using a transmission line network. Nature Communications 10, 434, doi:10.1038/s41467-018-08281-9 (2019).

29 Song, Z.-D., Lian, B., Regnault, N. \& Bernevig, B. A. Twisted bilayer graphene. II. Stable symmetry anomaly. Physical Review B 103, 205412, doi:10.1103/PhysRevB.103.205412 (2021).

30 Kang, J. \& Vafek, O. Non-Abelian Dirac node braiding and near-degeneracy of correlated phases at odd integer filling in magic-angle twisted bilayer graphene. Physical Review B 102, 035161, doi:10.1103/PhysRevB.102.035161 (2020).

31 Bouhon, A., Bzdušek, T. \& Slager, R.-J. Geometric approach to fragile topology beyond symmetry indicators. Physical Review B 102, 115135, doi:10.1103/PhysRevB.102.115135 (2020).

32 Lopes, P. L. e. S., Teo, J. C. Y. \& Ryu, S. Effective response theory for zero-energy Majorana bound states in three spatial dimensions. Physical Review B 91, 184111, doi:10.1103/PhysRevB.91.184111 (2015).

33 Ünal, F. N., Bouhon, A. \& Slager, R.-J. Topological Euler Class as a Dynamical Observable in Optical Lattices. Physical Review Letters 125, 053601, doi:10.1103/PhysRevLett.125.053601 (2020).

34 Rechtsman, M. C. et al. Photonic Floquet topological insulators. Nature 496, 196200, doi:10.1038/nature12066 (2013).

35 Hafezi, M., Mittal, S., Fan, J., Migdall, A. \& Taylor, J. M. Imaging topological edge states in silicon photonics. Nature Photonics 7, 1001-1005, doi:10.1038/nphoton.2013.274 (2013).

36 Dutt, A. et al. A single photonic cavity with two independent physical synthetic dimensions. Science 367, 59, doi:10.1126/science.aaz3071 (2020).

37 Ma, G., Xiao, M. \& Chan, C. T. Topological phases in acoustic and mechanical systems. Nature Reviews Physics 1, 281-294, doi:10.1038/s42254-019-0030-x (2019). 


\section{Table and Figures}

Table 1. Category of non-Abelian topological charges in four-band models. The three categories could be further decomposed into 10 conjugacy classes forming the generalized quaternion group $Q_{16}$. For the four-band system separated by three bandgaps, if we label each band with Zak phases of 0 or $\pi$, there are $2^{3}=8$ possibilities, corresponding to the 8 different eigenvalue sets. There two classes going beyond the Zak phase description ${ }^{9}$.

\begin{tabular}{|c|c|c|}
\hline$Q_{16}:$ Clifford-basis label & $Q_{16}:$ Band-index label & Eigenvalues: $\left(\lambda_{1}, \lambda_{2}, \lambda_{3}, \lambda_{4}\right)$ \\
\hline$\{+1\},\{-1\}$ & $\{+1\},\{-1\}$ & $(1,1,1,1)$ \\
$\left\{ \pm e_{1}\right\}$ & $\left\{ \pm q_{12}\right\}$ & $(-1,-1,1,1)$ \\
$\left\{ \pm e_{2}\right\}$ & $\left\{ \pm q_{13}\right\}$ & $(-1,1,-1,1)$ \\
$\left\{ \pm e_{3}\right\}$ & $\left\{ \pm q_{14}\right\}$ & $(-1,1,1,-1)$ \\
$\left\{ \pm e_{1} e_{2}\right\}$ & $\left\{ \pm q_{23}\right\}$ & $(1,-1,-1,1)$ \\
$\left\{ \pm e_{1} e_{3}\right\}$ & $\left\{ \pm q_{24}\right\}$ & $(1,-1,1,-1)$ \\
$\left\{ \pm e_{2} e_{3}\right\}$ & $\left\{ \pm q_{34}\right\}$ & $(1,1,-1,-1)$ \\
$\left\{+e_{1} e_{2} e_{3}\right\},\left\{-e_{1} e_{2} e_{3}\right\}$ & $\left\{+q_{1234}\right\},\left\{-q_{1234}\right\}$ & $(-1,-1,-1,-1)$ \\
\hline
\end{tabular}



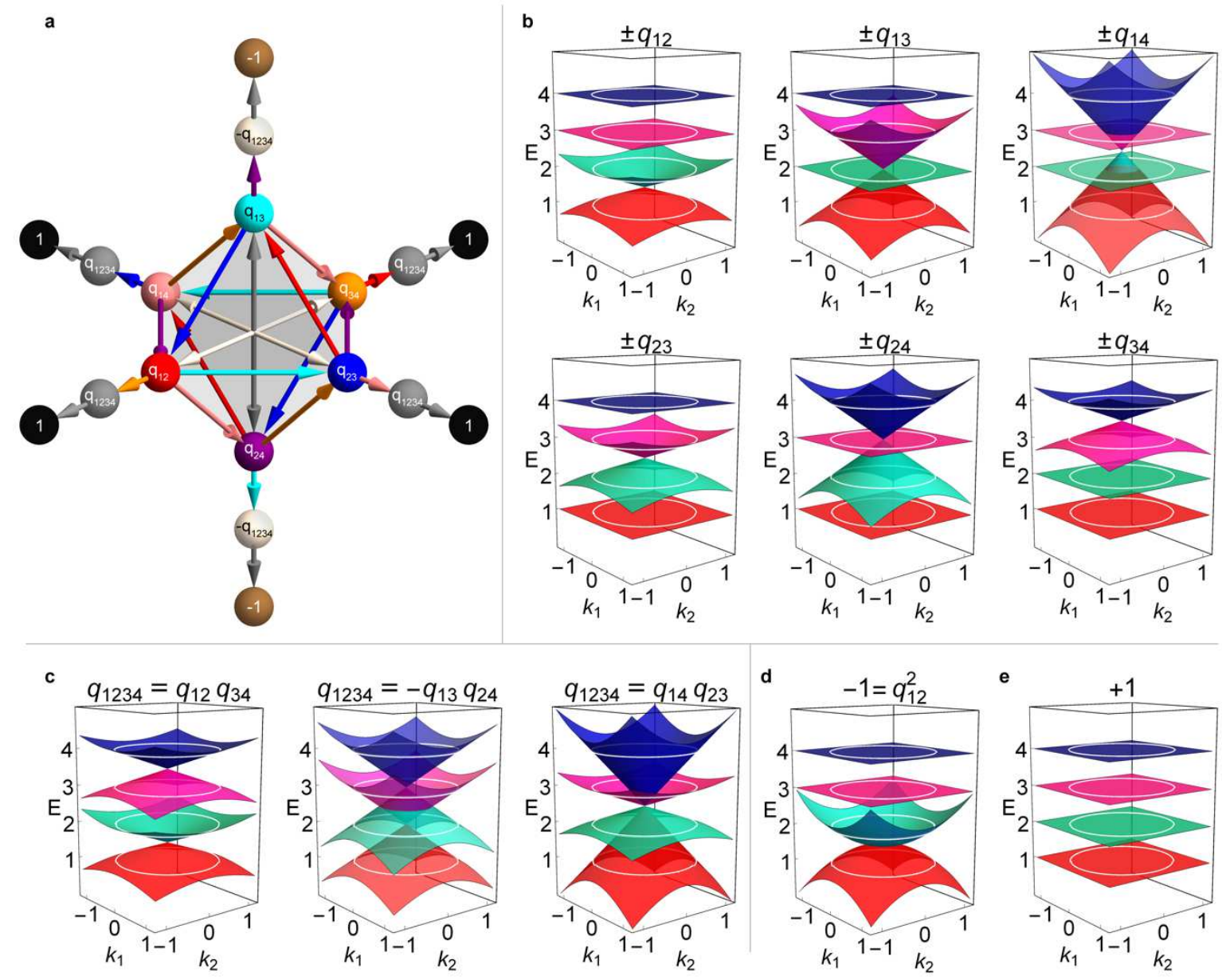

Figure 1. Non-Abelian topological charges in four-band models. a, Elements of the $Q_{16}$ group indicated by coloured spheres sitting on an outstretched regular octahedron and their mutual multiplications represented by the corresponding coloured arrows. For example, a red arrow $q_{12}$ brings a blue sphere $q_{23}$ to a cyan sphere $q_{13}$ indicating that $q_{23} q_{12}=q_{13}$. Full multiplication tables are provided in Supplementary Tables 1 and 2. b-e, The extended twodimensional bands corresponding to three different categories of the non-Abelian topological charges: $\pm q_{m n}, q_{1234}$ and \pm 1 , respectively. White circles indicate the corresponding $1 \mathrm{D}$ bands. The charge $q_{1234}$ can be decomposed into three ways in (c), which are all topologically equivalent. For charge -1 we take $-1=q_{12}^{2}$ as an example, and the other cases can be simply obtained by changing the linear Dirac cone degeneracies in panel (b) to be quadratic ones without any position shifting. 

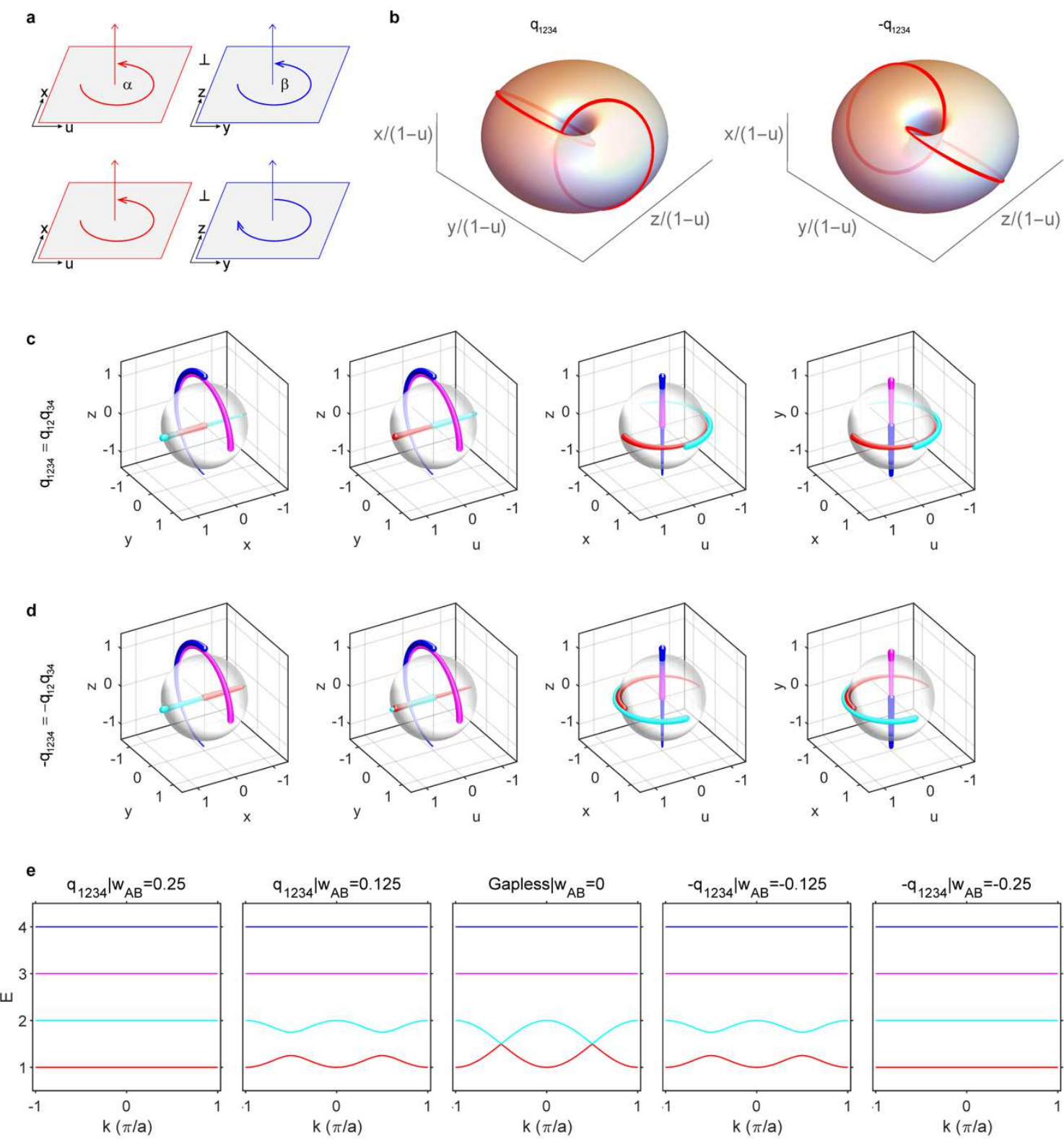

Figure 2. Non-Abelian topological charges $\pm \boldsymbol{q}_{1234}$ specific to four-band models. a, Rotations in four-dimension. For each rotation $R$, there is at least one pair of two orthogonal rotation-invariant planes, e.g. $A=o u x$ and $B=o y z$, which span the four-dimensional space. For any $\vec{a} \in A$ and $\vec{b} \in B$ we have $\vec{a} \perp \vec{b}, R \vec{a} \in A$ and $R \vec{b} \in B$. We define the angle between $\vec{a}$ and $R \vec{a}(\vec{b}$ and $R \vec{b})$ in the plane $A(B)$ as $\alpha(\beta)$. b, Four-dimensional Clifford tori $(u, x, y, z)$ are stereographically projected into $\mathbb{R}^{3}$ as the conventional tori $\left(\frac{x}{1-u}, \frac{y}{1-u}, \frac{z}{1-u}\right)$. The two linked circles represent the trajectory of one eigenstate (with applying all the four-dimensional $D_{2}$ rotations), the other three eigenstate trajectories overlap with this one. c and d, Orthographically projecting the four eigenstate trajectories (shown in different colours) onto four 3D solid spheres, where one component is hidden by projection in each sphere. e, The 
topological phase transition between charges $\pm q_{1234}$, other parameters are listed in Supplementary Table 4.

a

\begin{tabular}{|c|c|c|c|c|c|c|c|c|c|c|}
\hline $\mathrm{Q}_{16}$ & $\pm q_{12}$ & $\pm q_{13}$ & $\pm q_{14}$ & $\pm q_{23}$ & $\pm \mathrm{q}_{24}$ & $\pm q_{34}$ & +1 & -1 & $+q_{1234}$ & $-q_{1234}$ \\
\hline $4^{\text {th }}$ band & 0 & 0 & $\pm \pi$ & 0 & $\pm \pi$ & $\pm \pi$ & \multirow[t]{4}{*}{0} & \multirow[t]{4}{*}{$2 \pi$} & Two $+\pi$ & \multirow{4}{*}{$\begin{array}{l}\text { Two }+\pi \\
\text { Two } \mp \pi\end{array}$} \\
\hline $3^{\text {rd }}$ band & 0 & $\pm \pi$ & 0 & $\pm \pi$ & 0 & $\pm \pi$ & & & \multirow[t]{3}{*}{ Two $\pm \pi$} & \\
\hline $2^{\text {nd }}$ band & $\pm \pi$ & 0 & 0 & $\pm \pi$ & $\pm \pi$ & 0 & & & & \\
\hline $1^{\text {st }}$ band & $\pm \pi$ & $\pm \pi$ & $\pm \pi$ & 0 & 0 & 0 & & & & \\
\hline
\end{tabular}
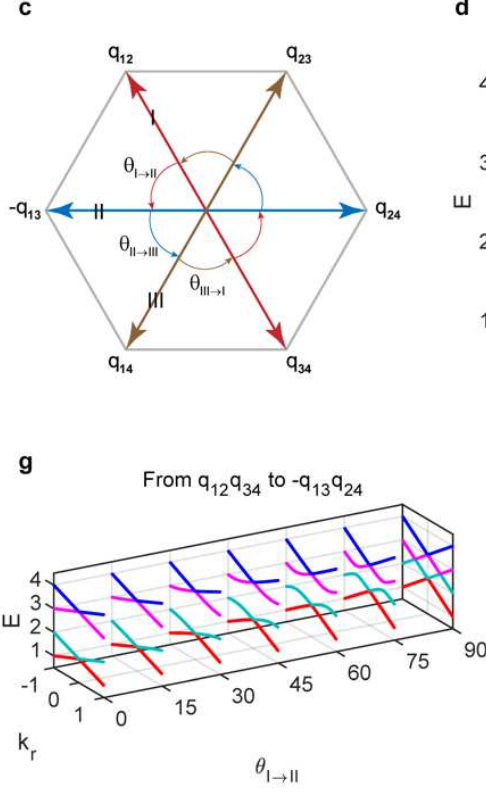

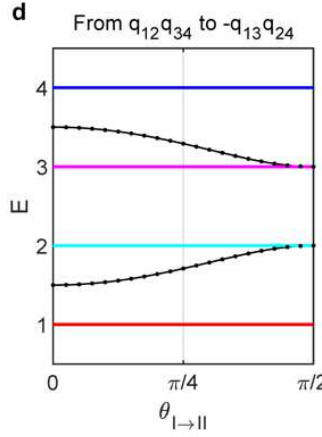

h

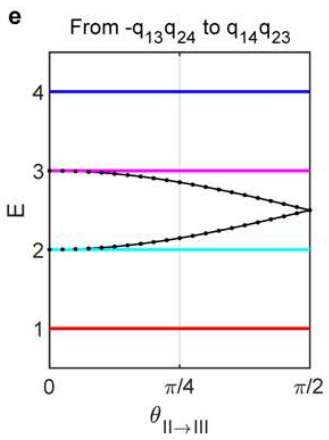

i

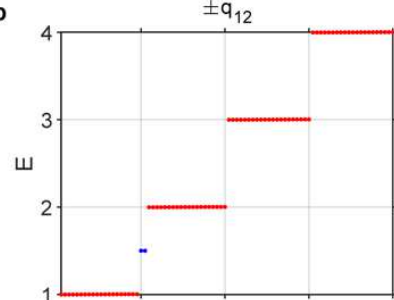

Index of Eigenstates
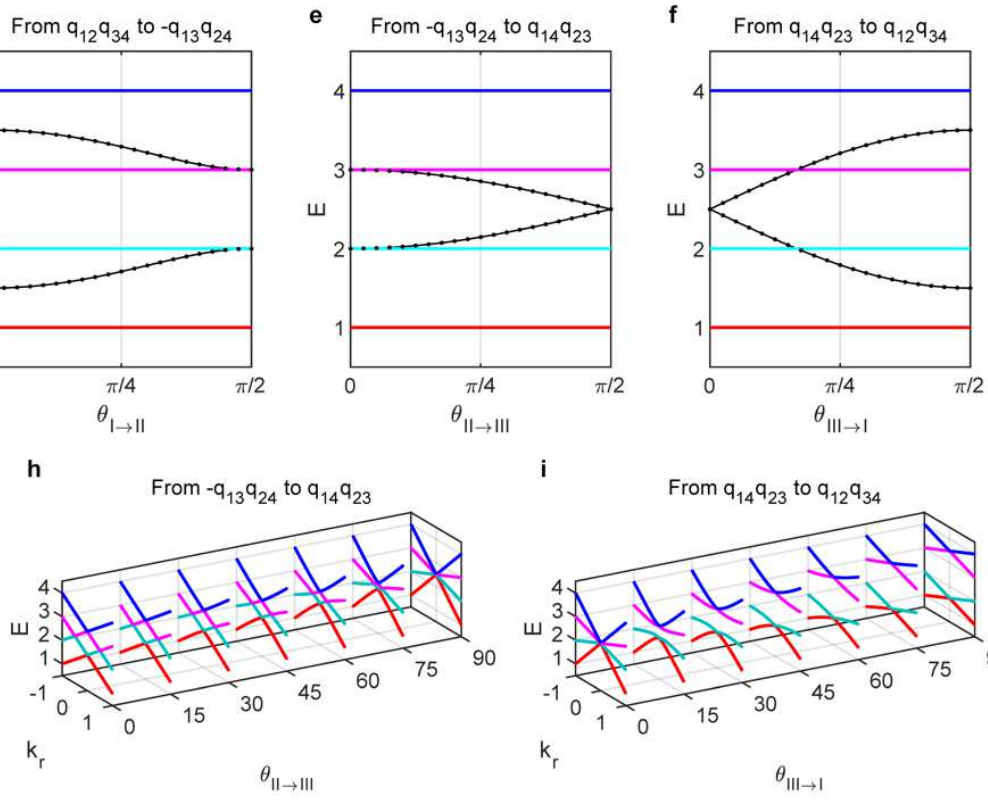

From $q_{14} q_{23}$ to $q_{12} q_{34}$

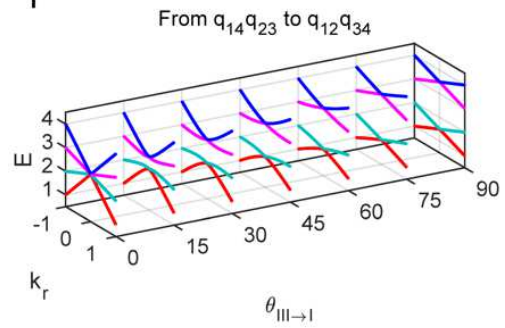

Figure 3. Zak phases and evolution of edge states. a, Zak phases for individual bands summarized for each non-Abelian topological charge. For charges $\pm q_{1234}$, two eigenvectors rotate $\pi$, while the other two rotate $+\pi$ or $-\pi$ depending on the factorizations (due to the handedness of subspace). b, Edge states of charges $\pm q_{12}$ occur at the bandgap sandwiched by the first and second bands. c, Schematic view of charge $q_{1234}$ factorizations and their mutual continuous transitions. The double-headed arrows indicate that the paired two factors commute, i.e. $q_{12} q_{34}=q_{34} q_{12}=q_{1234}$. The directional arcs define the continuous transitions parametrized by $\theta_{m \rightarrow n}$ with $m$ and $n$ taking values of I, II and III, corresponding to the factorizations of $q_{12} q_{34},-q_{13} q_{24}$ and $q_{14} q_{23}$, respectively. d-f, Evolution of edge states with varying the parameters $\theta_{m \rightarrow n}$, where lines/dots indicate the numerical/analytical results. $\mathbf{g}-\mathbf{i}$, Evolution of the extended two-dimensional bands corresponding to (d-f), respectively. Note that we only plot the radial cuts $E\left(k_{r}\right)$ as the $2 \mathrm{D}$ bands for ideal flat-band models are isotropic in the $\left(k_{1}, k_{2}\right)$ plane. 
a
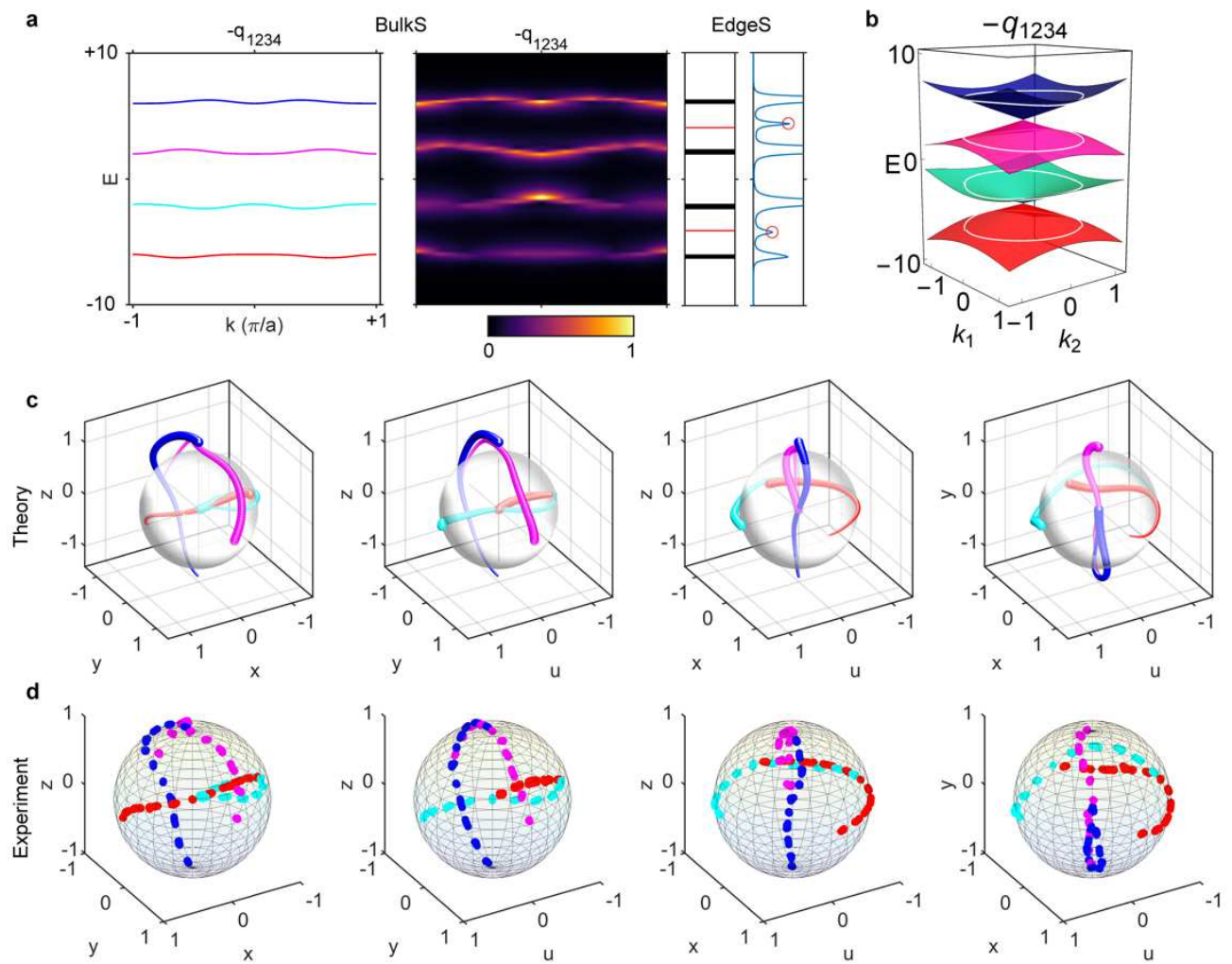

e

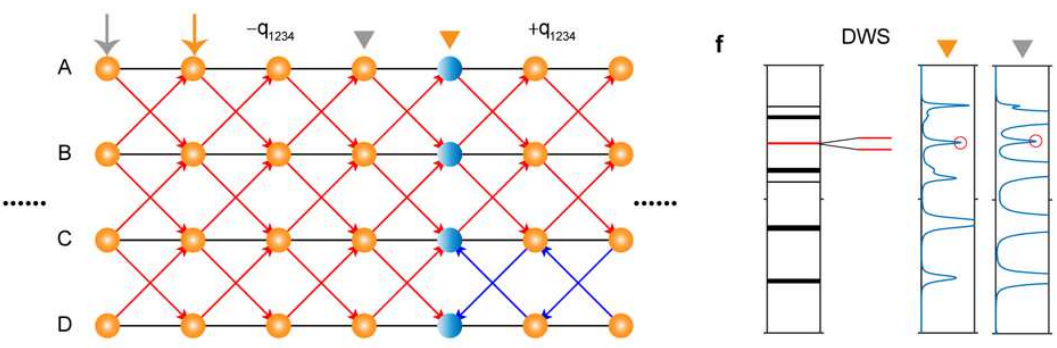

Figure 4. Experimental observation of the charge $-q_{1234}$ and edge/domain-wall states. a,

The left panels show the numerically calculated and experimentally measured energy bands of bulk states (BulkS). The right panels indicate the energy spectra probed at the hard boundary, where the red lines and the peaks marked by red circles represent the simulation and experiment results of edge states (EdgeS), respectively. b, The extended energy bands on a 2D plane, there is one linear Dirac cone between the first/third and second/fourth bands. White circles indicate the 1D energy bands. c/d, The calculated/measured orthographic projections of eigenstate trajectories. The colours of trajectories correspond to different bands in (a), respectively. The direction of linewidth decreasing indicates $k$ running from $-\pi$ to $\pi$. $\mathbf{e}$, The construction of domain-wall (marked by blue spheres) between charges $-q_{1234}$ and $q_{1234}$. The gray and yellow arrows/triangles denote two different excitation/probe positions. f, The calculated and measured energy spectra for the two different pairs of excitation and probe positions, 
respectively. The two domain-wall states are nearly degenerate. Detailed distribution of edge/domain-wall states is shown in Supplementary Figure 14/15.
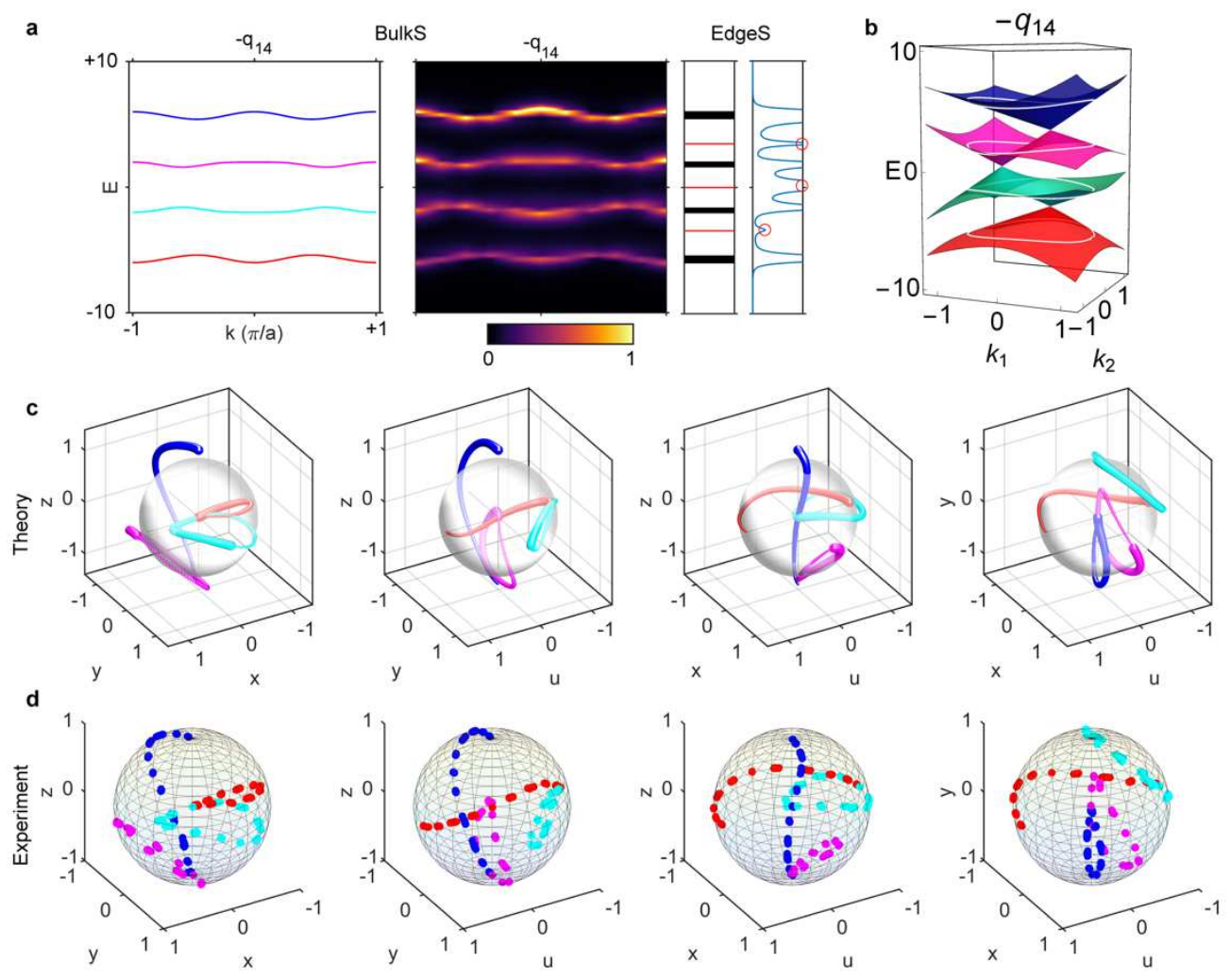

Figure 5. Experimental observation of the charge $-\boldsymbol{q}_{14}$ and edge states. a, The left panels show the numerically calculated and experimentally measured energy bands of bulk states (BulkS). The right panels indicate the energy spectra probed at the hard boundary, where the red lines and the peaks marked by red circles represent the simulation and experiment results of edge states (EdgeS), respectively. $\mathbf{b}$, The extended energy bands on a 2D plane, there is one linear Dirac cone in each bandgap. White circles indicate the 1D energy bands. c/d, The calculated/measured orthographic projections of eigenstate trajectories. The colours of trajectories correspond to different bands in (a), respectively. The direction of linewidth decreasing indicates $k$ running from $-\pi$ to $\pi$.

\section{Acknowledgements}

This work is supported by the Hong Kong RGC (AoE/P-02/12, 16310420), the Hong Kong Scholars Program (XJ2019007), the KAUST CRG grant (KAUST20SC01) and the Croucher foundation (CAS20SC01). 


\section{Supplementary Files}

This is a list of supplementary files associated with this preprint. Click to download.

- Supplementaryinformation.docx

- Supplementaryinformation.docx 